\title{
Completeness of the Bergman metric on non-smooth pseudoconvex domains
}

\author{
by Bo-Yong Chen (Shanghai)
}

\begin{abstract}
We prove that the Bergman metric on domains satisfying condition $\mathbf{S}$ is complete. This implies that any bounded pseudoconvex domain with Lipschitz boundary is complete with respect to the Bergman metric. We also show that bounded hyperconvex domains in the plane and convex domains in $\mathbb{C}^{n}$ are Bergman comlete.
\end{abstract}

1. Introduction. Let $D \subset \mathbb{C}^{n}$ be a bounded domain and let $K_{D}(z, w)$ be the Bergman kernel. The Bergman metric on $D$ is defined as follows:

$$
d s_{D}^{2}=\sum_{j, k=1}^{n} \frac{\partial^{2} \log K_{D}(z, z)}{\partial z_{j} \partial \bar{z}_{k}} d z_{j} d \bar{z}_{k} .
$$

In [11] Kobayashi posed an interesting question:

Which bounded pseudoconvex domains in $\mathbb{C}^{n}$ are complete with respect to the Bergman metric?

The assumption of pseudoconvexity is necessary (cf. [4]). It is quite clear that any bounded pseudoconvex domain with $C^{\infty}$-boundary is complete w.r.t. $d s_{D}^{2}$ (cf. [14]). In [13] Ohsawa has proved that the Bergman metric of any pseudoconvex domain with $C^{1}$-boundary is complete. In this article, we first study a class of pseudoconvex domains defined as follows:

Definition. We say that a domain $D$ in $\mathbb{C}^{n}$ satisfies condition $\mathbf{S}$ if there exists a sequence $\left\{D_{j}\right\}$ of pseudoconvex domains with $D \subset \subset D_{j}$ such that

(1) $\Lambda_{j}=\sup _{z \in \partial D} d_{D_{j}}(z) \rightarrow 0$ as $j \rightarrow \infty$, where $d_{D_{j}}(z)=d\left(z, \partial D_{j}\right)$ is the Euclidian distance from $z$ to $\partial D_{j}$;

(2) there exist reals $r \geq 1$ and $0<\alpha \leq 1$ such that $\Lambda_{j} \leq r \lambda_{j}^{\alpha}$, where $\lambda_{j}=\inf _{z \in \partial D} d_{D_{j}}(z)$.

1991 Mathematics Subject Classification: Primary 32H10.

Key words and phrases: Bergman metric. 
We say that $D$ satisfies condition $\mathbf{S}$ locally if for each $z^{0} \in \partial D$, there exists a ball $B\left(z^{0}, r_{0}\right)$ such that $D \cap B\left(z^{0}, r_{0}\right)$ satisfies condition $\mathbf{S}$.

One can easily conclude that $D$ is pseudoconvex if $D$ satisfies condition S locally.

We have the following main result:

TheOREM 1.1. Let $D$ be a domain which satisfies condition $\mathbf{S}$ locally. Then $D$ is complete w.r.t. the Bergman metric.

From Theorem 1.1 we can deduce

Theorem 1.2. The Bergman metric of any bounded pseudoconvex domain with Lipschitz boundary is complete.

It is known from [5] that any bounded pseudoconvex domain with Lipschitz boundary is hyperconvex, that is, it admits a bounded continuous plurisubharmonic (psh) exhaustion function. Naturally, one would ask:

(a) Is any bounded hyperconvex domain in $\mathbb{C}^{n}$ complete w.r.t. the Bergman metric?

(b) Are the hyperconvexity and the Bergman completeness equivalent?

In Section 5 we will construct a domain which is not hyperconvex but complete w.r.t. the Bergman metric. Question (a) seems to be quite difficult. However, we can prove that the answer is affirmative for some special cases.

Given a domain $D \subset \mathbb{C}^{n}$ and $\zeta \in D$, we consider the function

$u_{D}(\zeta, z)=u_{\zeta}(z)=\sup \{v(z) \mid v$ is psh on $D$,

$$
v<0 \text { and } v(w) \leq \log |w-\zeta|+O(1), w \in D\},
$$

which is the pluricomplex Green function on $D$ with logarithmic pole at $\zeta$ (cf. [1], [5]). We obtain another main result:

Theorem 1.3. Let $D$ be a bounded hyperconvex domain in $\mathbb{C}^{n}$ and suppose that $u_{D}(\zeta, z)$ is symmetric. Then the Bergman metric on $D$ is complete.

Since the complex Green function of hyperconvex domains in $\mathbb{C}$ is symmetric (cf. [12]), Theorem 1.3 implies

Corollary 1.4. Any bounded hyperconvex domain in $\mathbb{C}$ is complete w.r.t. the Bergman metric.

It is known from [3] that any bounded convex domain in $\mathbb{C}^{n}$ is hyperconvex, and the pluricomplex Green function is symmetric (cf. [15]). Immediately we obtain

COROllary 1.5. The Bergman metric of any bounded convex domain in $\mathbb{C}^{n}$ is complete. 
The proofs of the theorems are based on the techniques of $L^{2}$-estimates for the $\bar{\partial}$-equation on complete Kähler manifolds due to Diederich and Ohsawa.

2. Preliminaries. Let $X$ be a complete manifold of dimension $n$ and $d s^{2}$ be a Hermitian metric on $X$. Let $C_{0}^{p, q}(X)$ be the set of $C^{\infty}$-differentiable $(p, q)$-forms on $X$ with compact support, and let $\varphi: X \rightarrow \mathbb{R}$ be a continuous function. We define an inner product by

$$
(u, v)_{d s^{2}, \varphi}:=\int_{X} e^{-\varphi} u \wedge *_{d s^{2}} \bar{v}
$$

for $u, v \in C_{0}^{p, q}(X)$. Here $* d s^{2}$ denotes the Hodge star operator associated with $d s^{2}$. Put

$$
\|u\|_{d s^{2}, \varphi}:=(u, u)_{d s^{2}, \varphi}^{1 / 2}
$$

and denote by $L^{p, q}\left(X, d s^{2}, \varphi\right)$ the space of all square integrable $(p, q)$-forms on $X$, i.e., the completion of $C_{0}^{p, q}(X)$ with respect to the norm $\|\cdot\|_{d s^{2}, \varphi}$. Now we recall the following useful result:

Proposition 2.1 (cf. Theorem 3 in [6]). Let $X$ be a complex manifold that admits a complete Kähler metric with a positive $C^{\infty}$ global potential function $\eta$, and let $\psi: X \rightarrow \mathbb{R}$ be another $C^{\infty}$ strictly psh function on $X$ satisfying the estimate $\partial \bar{\partial} \psi \geq \partial \psi \bar{\partial} \psi$. Furthermore, let $\varphi$ be any $C^{\infty}$ psh function on $X$. Then, for any $\bar{\partial}$-closed $(n, 1)$-form $g$ on $X$ satisfying $\|g\|_{\partial \bar{\partial} \psi, \varphi}<\infty$, there is a measurable $(n, 0)$-form $h$ satisfying $\bar{\partial} h=g$ and $\|h\|_{\varphi} \leq C\|g\|_{\partial \bar{\partial} \psi, \varphi}$, where $C$ is a numerical constant (independent of $X, \psi, \varphi, g)$ and $\|h\|_{\varphi}^{2}=$ $\left|\int_{X} e^{-\varphi} h \wedge \bar{h}\right|$.

3. Proofs of Theorems 1.1-1.2. Let $D$ be a bounded domain in $\mathbb{C}^{n}$. We denote by $H^{2}(D)$ the space of all square integrable holomorphic functions on $D$ and by $H(\bar{D})$ the space of all functions holomorphic in a neighbourhood of $\bar{D}$. The $L^{2}$-norm is denoted by $\|\cdot\|_{D}$, and $\|f\|_{D, \varphi}$ means the $L^{2}$-norm of $f$ with weight $e^{-\varphi}$, where $\varphi$ is a continuous real function on $D$. We claim

Lemma 3.1. Let $D$ be a domain satisfying condition $\mathbf{S}$. Then $H(\bar{D})$ is dense in $H^{2}(D)$.

Proof. Let $f \in H^{2}(D)$. Without loss of generality we can assume $\|f\|_{D} \leq 1$. We define $D_{j, t}=\left\{z \in D_{j} \mid d_{D_{j}}(z)>t\right\}, t>0$. Then $D_{j, t}$ is pseudoconvex since $-\log d_{D_{j}}(z) / t$ is a psh exhaustion function on $D_{j, t}$, and we have

$$
D_{j, \Lambda_{j}} \subset D \subset \subset D_{j, t} \quad \text { for all } t<\lambda_{j} .
$$


Put

$$
\beta= \begin{cases}1 / 2, & \alpha=1, \\ \frac{1-\alpha}{1-\alpha / 2}, & 0<\alpha<1 .\end{cases}
$$

Let $\chi: \mathbb{R} \rightarrow[0,1]$ be a $C^{\infty}$ function satisfying $\left.\chi\right|_{(-\infty, 1+1 /(2 \log \beta))}=1$ and $\left.\chi\right|_{[1, \infty)}=0$. Since each continuous psh function can be approximated by a decreasing sequence of $C^{\infty}$ strictly psh functions, there exist for each $j$ a number $\nu_{j}$ with $\lambda_{j} / 2<\nu_{j}<\lambda_{j}$ and a $C^{\infty}$ strictly psh function $u_{j}$ defined on $D_{j, \nu_{j}}$ such that

(1) $-\log \frac{d_{D_{j}}(z)}{\lambda_{j} / 2}<u_{j}(z)<0$ on $D_{j, \nu_{j}}$,

(2) $u_{j}(z)+\log \frac{d_{D_{j}}(z)}{\lambda_{j} / 2}<\left(\beta^{-1}-\beta^{-1 / 2}\right) \log \frac{\Lambda_{j}}{\lambda_{j} / 2}, \forall z \in D$.

We write $\widetilde{D}_{j}=D_{j, \nu_{j}}$ and put

$$
\psi_{j}=-\log \left(-u_{j}\right), \quad \varrho_{j}=\chi\left(\psi_{j}+\log \log \frac{\Lambda_{j}}{\lambda_{j} / 2}+1\right) .
$$

Then $\psi_{j}$ is a $C^{\infty}$ strictly psh function on $\widetilde{D}_{j}$ satisfying

$$
\partial \bar{\partial} \psi_{j} \geq \partial \psi_{j} \bar{\partial} \psi_{j}
$$

This gives

$$
\left|\bar{\partial} \varrho_{j}\right|_{\partial \bar{\partial} \psi_{j}} \leq \sup \left|\chi^{\prime}\right|
$$

where $\left|\bar{\partial} \varrho_{j}\right|_{\partial \bar{\partial} \psi_{j}}$ is the point-norm of $\bar{\partial} \varrho_{j}$ w.r.t. the metric $\partial \bar{\partial} \psi_{j}$.

From (1) we obtain

$$
\operatorname{supp} \varrho_{j} \subset D_{j, \Lambda_{j}} \subset D .
$$

So we can define a $C^{\infty} \bar{\partial}$-closed $(n, 1)$-form on $\widetilde{D}_{j}$ as follows:

$$
g_{j}=\bar{\partial}\left(\varrho_{j} f\right) \wedge d z_{1} \wedge \ldots \wedge d z_{n} .
$$

Since

where

$$
\begin{aligned}
\operatorname{supp} \bar{\partial} \varrho_{j} & \subset\left\{z \in D \mid u_{j}(z)>-\beta^{-1 / 2} \log \frac{\Lambda_{j}}{\lambda_{j} / 2}\right\} \\
& \subset\left\{z \in D \mid-\log \frac{d_{D_{j}}(z)}{\lambda_{j} / 2}>-\beta^{-1} \log \frac{\Lambda_{j}}{\lambda_{j} / 2}\right\} \\
& =D \backslash D_{j, \mu_{j}},
\end{aligned}
$$

$$
\mu_{j}= \begin{cases}2 r \Lambda_{j}, & \alpha=1, \\ \left(2^{\alpha} r\right)^{1 /(2(1-\alpha))} \Lambda_{j}^{1 / 2}, & 0<\alpha<1,\end{cases}
$$


we obtain

$$
\left\|g_{j}\right\|_{\partial \bar{\partial} \psi_{j}, 0}^{2}=2^{n} \int_{\widetilde{D}_{j}}\left|\bar{\partial} \varrho_{j}\right|_{\partial \bar{\partial} \psi_{j}}^{2}|f|^{2} d V_{n} \leq 2^{n} \sup \left|\chi^{\prime}\right|^{2}\|f\|_{D \backslash D_{j, \mu_{j}}}^{2},
$$

where $d V_{k}$ denotes the $2 k$-dimensional Lebesgue measure.

Since any pseudoconvex domain admits a complete Kähler metric with a positive global potential (cf. [7], p. 49), by Proposition 2.1 there exists an $(n, 0)$-form $h_{j}=\widetilde{h}_{j} d z_{1} \wedge \ldots \wedge d z_{n}$ on $\widetilde{D}_{j}$ satisfying $\bar{\partial} h_{j}=g_{j}$ and

$$
\left\|\widetilde{h}_{j}\right\|_{\widetilde{D}_{j}} \leq C \sup \left|\chi^{\prime}\right| \cdot\|f\|_{D \backslash D_{j, \mu_{j}}},
$$

where $C$ is a numerical constant (independent of $f, j, \alpha, D$ ).

Hence $f_{j}=\varrho_{j} f-\widetilde{h}_{j}$ is holomorphic on $\widetilde{D}_{j}$ and satisfies

$$
\left\|f_{j}-f\right\|_{D} \leq\left\|\left(1-\varrho_{j}\right) f\right\|_{D}+\left\|\widetilde{h}_{j}\right\|_{D} \leq(1+C) \sup \left|\chi^{\prime}\right| \cdot\|f\|_{D \backslash D_{j, \mu_{j}}} .
$$

Given any $\varepsilon>0$, there exists a $\delta=\delta(\varepsilon)>0$ such that

$$
\|f\|_{D \backslash D_{\delta}}<\varepsilon
$$

where $D_{t}=\left\{z \in D \mid d_{D}(z)>t\right\}, t>0$. Since $\Lambda_{j} \rightarrow 0$ as $j \rightarrow \infty$, one has $\mu_{j}<\delta / 2$ for all sufficiently large $j$. This gives $D_{j, \mu_{j}} \supset D_{j, \delta / 2}$ for all sufficiently large $j$. Since $d_{D_{j}}(z) \rightarrow d_{D}(z)$ on $D$, there exists a $j(\varepsilon)$ such that

Thus

$$
D_{j(\varepsilon), \mu_{j(\varepsilon)}} \supset D_{\delta}
$$

$$
\left\|f_{j(\varepsilon)}-f\right\|_{D} \leq(1+C) \varepsilon \sup \left|\chi^{\prime}\right| .
$$

The proof is complete.

Lemma 3.2. $\lim _{z \rightarrow \partial D} K_{D}(z, z)=\infty$ if $D$ is a domain which satisfies condition $\mathbf{S}$ locally.

Proof. We can easily conclude that $D$ satisfies the cone condition in the sense of Pflug (cf. [14], p. 399), hence we have $\lim _{z \rightarrow \partial D} K_{D}(z, z)=\infty$.

The following localization lemma for the Bergman metric is perhaps known:

Lemma 3.3. Let $D$ be a bounded pseudoconvex domain in $\mathbb{C}^{n}$ and $z^{0}$ be any point in $\partial D$. Suppose that $U, V$ are two open neighbourhoods of $z^{0}$ with $V \subset \subset U$. Then

$$
d s_{D}^{2}(\zeta ; X) \geq C d s_{D \cap U}^{2}(\zeta ; X), \quad \forall X \in T^{1,0}\left(\mathbb{C}^{n}\right), \quad \zeta \in V \cap D,
$$

where $C$ is a constant (independent of $\zeta, X$ ).

Proof. From the original work of Bergman (cf. [2]) we know that for any $X \in T^{1,0}\left(\mathbb{C}^{n}\right)$ and $\zeta \in D$ we have

$$
d s_{D}^{2}(\zeta ; X)=K_{D}^{-1}(\zeta, \zeta) \sup \left\{|X f|^{2} \mid f \in H^{2}(D),\|f\|_{D} \leq 1, f(\zeta)=0\right\} .
$$


We choose a $C^{\infty}$ function $\eta: \mathbb{C}^{n} \rightarrow[0,1]$ such that $\eta=1$ on a neighbourhood of $\bar{V}, \eta=0$ on $\mathbb{C}^{n} \backslash U$. Fix any point $\zeta \in D \cap V$ and for any $f \in H^{2}(D \cap U)$ with $\|f\|_{D \cap U} \leq 1$ and $f(\zeta)=0$ put $v=\bar{\partial}(\eta f)$. Thus we have defined a $C^{\infty} \bar{\partial}$-closed $(0,1)$-form on $D$. Let $\psi(z)=2(n+1) \log |z-\zeta|$. We have

$$
\int_{D}|v|^{2} e^{-\psi} d V_{n} \leq C_{1}
$$

where $C_{1}$ is a constant (independent of $\zeta, f$ ). By a well known Hörmander theorem, there exists a function $u(z)$ which satisfies $\bar{\partial} u=v$ and

$$
\int_{D}|u|^{2}|z-\zeta|^{-2(n+1)} d V_{n} \leq C_{2},
$$

where $C_{2}$ is a constant (independent of $\left.\zeta, f\right)$. Then $F(z)=\eta(z) f(z)-u(z)$ is a holomorphic function on $D$ and $\|F\|_{D} \leq 1+C_{3}$, where $C_{3}$ is also independent of $\zeta, f$. Since $\eta=1$ on $V, u$ is holomorphic on $V$ and we have

$$
u(\zeta)=0, \quad \frac{\partial u}{\partial z_{j}}(\zeta)=0, \quad \forall 1 \leq j \leq n .
$$

So we have

$$
F(\zeta)=-u(\zeta)=0, \quad \frac{\partial F}{\partial z_{j}}(\zeta)=\frac{\partial f}{\partial z_{j}}(\zeta), \quad \forall 1 \leq j \leq n .
$$

Hence

$$
d s_{D}^{2}(\zeta ; X) \geq C d s_{D \cap U}^{2}(\zeta ; X), \quad \forall X \text { and } \zeta \in D \cap V,
$$

where $C=\left(1+C_{3}\right)^{-2}$.

Theorem 1.1 is immediately derived from Lemmas $3.1-3.3$ and the following proposition:

Proposition 3.4 (cf. [9]). Let $D$ be a bounded domain in $\mathbb{C}^{n}$ and assume

(1) the bounded holomorphic functions in $D$ are dense in $H^{2}(D)$;

(2) $\lim _{z \rightarrow \partial D} K_{D}(z, z)=\infty$.

Then the Bergman metric of $D$ is complete.

REMARK. In fact, we can replace assumption (1) by a weaker condition:

For each $z^{0} \in \partial D$ and $f \in H^{2}(D), f$ can be approximated in $L^{2}$-norm by a family of holomorphic functions on $D$ which are bounded in some neighbourhood of $z^{0}$.

This can be proved easily with the method of [14] (cf. p. 409).

Proof of Theorem 1.2. Let $D$ be a bounded pseudoconvex domain with Lipschitz boundary. For each $z^{0} \in \partial D$, there exist reals $\varepsilon_{0}, r_{0}>0$ and a vector $T_{0}$ that points outside of $D$ such that $z+\varepsilon T_{0} \in \mathbb{C}^{n} \backslash \bar{D}$ for all $z \in\left(\mathbb{C}^{n} \backslash \bar{D}\right) \cap B\left(z^{0}, r_{0}\right)$ and $0<\varepsilon<\varepsilon_{0}$. 
It is known from [5] that $D$ admits a $C^{\infty}$ strictly psh function $u$ on $D$ such that $u<0$ on $D$ and $\lim _{z \rightarrow \partial D} u(z)=0$. For $\varepsilon, r>0$, we set

$$
D_{r}^{\varepsilon}=\left\{z \in B\left(z^{0}, r\right) \mid u\left(z-\varepsilon T_{0}\right)<0\right\} .
$$

Put $D_{\varepsilon}=D_{r_{0} / 2+\varepsilon}^{\varepsilon}$ for $\varepsilon<\min \left\{\varepsilon_{0}, r_{0} / 4\right\}$. Then:

(1) $D_{\varepsilon}$ is pseudoconvex;

(2) $D \cap B\left(z^{0}, r_{0} / 2\right) \subset \subset D_{\varepsilon}$;

(3) there exists a constant $0<C<1$ such that

$$
C \varepsilon<d_{D_{\varepsilon}}(z)<\varepsilon
$$

for all $z \in \partial\left(D \cap B\left(z^{0}, r_{0} / 2\right)\right)$. Hence $D$ locally satisfies condition $\mathbf{S}$, and therefore Theorem 1.2 is a direct consequence of Theorem 1.1.

4. Bergman metric on hyperconvex domains. Let $D$ be a bounded hyperconvex domain in $\mathbb{C}^{n}$, and $u_{D}(\zeta, z)$ be the pluricomplex Green function on $D$. From [10] we know that there exists a $C^{\infty}$ strictly psh function $u$ on $D$ such that $u<0$ on $D$ and $\lim _{z \rightarrow \partial D} u(z)=0$. We set $D_{t}=\{z \in D \mid$ $u(z)<t\}$. The following fact is due to Demailly:

Proposition 4.1 (cf. [5], p. 531). For each $\zeta \in D, u_{D}(\zeta, \cdot)$ is a continuous psh exhaustion function on $D$ with values in $[-\infty, 0)$ and $u_{D}(\zeta, z) \sim$ $\log |z-\zeta|$ as $z \rightarrow \zeta$.

Now we prove the following two lemmas:

Lemma 4.2. Suppose that $D$ is a bounded hyperconvex domain in $\mathbb{C}^{n}$. Then for each $\zeta \in D$ and $f \in H^{2}(D)$, there exists a function $F \in H^{2}(D)$ satisfying $F(\zeta)=0$ and

$$
\|F-f\|_{D} \leq C\|f\|_{D_{\zeta,-1}},
$$

where $D_{\zeta, t}=\left\{z \in D \mid u_{D}(\zeta, z)<t\right\}$ and $C$ is a constant (independent of $\zeta, f)$.

Proof. Fix $\zeta$. There are negative $C^{\infty}$ strictly psh functions $u_{\varepsilon}$ defined on $D_{-a(\varepsilon)}$, where $a(\varepsilon) \rightarrow 0$ as $\varepsilon \rightarrow 0$, such that $u_{\varepsilon} \downarrow u_{D}(\zeta, \cdot)$ as $\varepsilon \downarrow 0$.

Let $0 \leq \kappa \leq 1$ be a $C^{\infty}$ function on $\mathbb{R}$ such that $\kappa(t)=1$ on $(-\infty$, $1-\log 2)$ and $\kappa(t)=0$ on $[1, \infty)$. Put

$$
\psi_{\varepsilon}=-\log \left(-u_{\varepsilon}\right), \quad \varrho_{\varepsilon}(z)=1-\kappa\left(\psi_{\varepsilon}(z)+1\right) .
$$

Again we have

$$
\left|\bar{\partial} \varrho_{\varepsilon}\right|_{\partial \bar{\partial} \psi_{\varepsilon}} \leq \sup \left|\kappa^{\prime}\right| .
$$


Put $g_{\varepsilon}=\bar{\partial}\left(\varrho_{\varepsilon} f\right) \wedge d z_{1} \wedge \ldots \wedge d z_{n}$. One has

$$
\begin{aligned}
\left\|g_{\varepsilon}\right\|_{\partial \bar{\partial} \psi_{\varepsilon}, 2 n u_{\varepsilon}}^{2} & \leq 2^{n} \sup \left|\kappa^{\prime}\right|^{2} \int_{-2<u_{\varepsilon}<-1}|f|^{2} e^{-2 n u_{\varepsilon}} d V_{n} \\
& \leq 2^{n} e^{4 n} \sup \left|\kappa^{\prime}\right|^{2}\|f\|_{D_{\zeta,-1}}^{2}
\end{aligned}
$$

since $u_{\varepsilon} \geq u_{D}(\zeta, \cdot)$. By Proposition 2.1, there exists an $(n, 0)$-form $h_{\varepsilon}=$ $\widetilde{h}_{\varepsilon} d z_{1} \wedge \ldots \wedge d z_{n}$ on $D_{-a(\varepsilon)}$ such that $\bar{\partial} h_{\varepsilon}=g_{\varepsilon}$ and

$$
\left\|\widetilde{h}_{\varepsilon}\right\|_{D_{-a(\varepsilon)}, 2 n u_{\varepsilon}} \leq C_{0}\|f\|_{D_{\zeta,-1}},
$$

where $C_{0}$ is a constant (independent of $\zeta, \varepsilon, f$ ). Then $F_{\varepsilon}=\varrho_{\varepsilon} f-\widetilde{h}_{\varepsilon}$ is holomorphic on $D_{-a(\varepsilon)}$ and

$$
\left\|F_{\varepsilon}-f\right\|_{D_{-a(\varepsilon)}} \leq\left\|\left(1-\varrho_{\varepsilon}\right) f\right\|_{D_{-a(\varepsilon)}}+\left\|\widetilde{h}_{\varepsilon}\right\|_{D_{-a(\varepsilon)}, 2 n u_{\varepsilon}} \leq\left(1+C_{0}\right)\|f\|_{D_{\zeta,-1}} .
$$

We also have

$$
\left\|F_{\varepsilon}\right\|_{D_{-a(\varepsilon)}, 2 n u_{\varepsilon}} \leq\left\|\varrho_{\varepsilon} f\right\|_{D_{-a(\varepsilon)}, 2 n u_{\varepsilon}}+\left\|\widetilde{h}_{\varepsilon}\right\|_{D_{-a(\varepsilon)}, 2 n u_{\varepsilon}} \leq\left(e^{2 n}+C_{0}\right)\|f\|_{D} .
$$

Since $u_{\varepsilon}<0$, we can choose a sequence $\varepsilon_{j} \rightarrow 0$ such that $F_{\varepsilon_{j}}$ converges weakly to a holomorphic function $F$ on $D$. Hence

$$
\|F-f\|_{D} \leq\left(1+C_{0}\right)\|f\|_{D_{\zeta,-1}} .
$$

Since $u_{\varepsilon}$ decreases with $\varepsilon$, for each $b>0$ and $\varepsilon$ with $0<a(\varepsilon)<b$ we have

$$
\|F\|_{D_{-b}, 2 n u_{\varepsilon}} \leq\left(C_{0}+e^{2 n}\right)\|f\|_{D} .
$$

Letting $\varepsilon \rightarrow 0$ and then $b \rightarrow 0$, one gets

$$
\|F\|_{D, 2 n u_{D}(\zeta, \cdot)} \leq\left(C_{0}+e^{2 n}\right)\|f\|_{D} .
$$

Since $u_{D}(\zeta, z) \sim \log |z-\zeta|$ as $z \rightarrow \zeta$, one has $F(\zeta)=0$.

Lemma 4.3. Suppose that $D$ is a bounded hyperconvex domain in $\mathbb{C}^{n}$, and $u_{D}(\zeta, z)$ is symmetric. Then the volume of $D_{\zeta,-1}$ tends to zero as $\zeta \rightarrow \partial D$.

Proof. The proof is due to Ohsawa (cf. [12]). For any $\varepsilon>0$, there exists a $C^{\infty}$ function $\kappa_{\varepsilon}(z)$ such that $\operatorname{supp} \kappa_{\varepsilon} \subset D_{-\varepsilon / 2}$ and $\left.\kappa_{\varepsilon}\right|_{D_{-\varepsilon}}=1$. Then we can find a constant $C=C(\varepsilon)$ such that $C u(z)+\kappa_{\varepsilon}(z) \log |z-\zeta|$ is a negative psh function on $D$ for all $\zeta \in D_{-\varepsilon}$. Hence there exists a $\delta>0$ such that

$$
u_{D}(\zeta, z)>-1, \quad \forall \zeta \in D_{-\varepsilon}, z \in D \backslash D_{-\delta} .
$$

Since $u_{D}(\zeta, z)=u_{D}(z, \zeta)$, for any $\varepsilon>0$ there exists a $\delta>0$ such that

$$
u_{D}(\zeta, z)>-1, \quad \forall \zeta \in D \backslash D_{-\delta}, z \in D_{-\varepsilon} .
$$

This proves the lemma.

To prove Theorem 1.3, we need the following proposition:

Proposition 4.4 (cf. [14], p. 408). Let $D$ be a bounded domain in $\mathbb{C}^{n}$, and $\left\{\zeta^{j}\right\}_{j=1}^{\infty} \subset D$ a Cauchy sequence w.r.t. the Bergman metric. Then there 
exist a subsequence $\left\{\zeta^{j_{k}}\right\}_{k=1}^{\infty}$ and real numbers $\theta_{k}$ such that

$$
\left\{\frac{K_{D}\left(\cdot, \zeta^{j_{k}}\right)}{K_{D}^{1 / 2}\left(\zeta^{j_{k}}, \zeta^{j_{k}}\right)} e^{i \theta_{k}}\right\}_{k=1}^{\infty}
$$

is a Cauchy sequence in $H^{2}(D)$.

Proof of Theorem 1.3. Suppose that $D$ is not complete w.r.t. the Bergman metric. Then there exists a Cauchy sequence $\left\{\zeta^{j}\right\}_{j=1}^{\infty}$ w.r.t. the Bergman metric which converges to a boundary point $\zeta^{0}$ of $D$ as $j \rightarrow \infty$. Proposition 4.4 gives us a subsequence $\left\{\zeta^{j_{k}}\right\}_{k=1}^{\infty}$ and real numbers $\theta_{k}$ satisfying

$$
\frac{K_{D}\left(\cdot, \zeta^{j_{k}}\right)}{K_{D}^{1 / 2}\left(\zeta^{j_{k}}, \zeta^{j_{k}}\right)} e^{i \theta_{k}} \rightarrow f
$$

in $H^{2}(D)$ with $\|f\|_{D}=1$. This yields

$$
\frac{\left|f\left(\zeta^{j_{k}}\right)\right|}{K_{D}^{1 / 2}\left(\zeta^{j_{k}}, \zeta^{j_{k}}\right)}=\left|e^{-i \theta_{k}}\left(f, \frac{K_{D}\left(\cdot, \zeta^{j_{k}}\right)}{K_{D}^{1 / 2}\left(\zeta^{j_{k}}, \zeta^{j_{k}}\right)}\right)\right| \rightarrow\|f\|^{2}=1 .
$$

By Lemma 4.2 there exists a sequence $\left\{F_{k}\right\}_{k=1}^{\infty}$ of holomorphic functions on $D$ satisfying $F_{k}\left(\zeta^{j_{k}}\right)=0$ and

$$
\left\|F_{k}-f\right\|_{D} \leq C\|f\|_{D_{\zeta^{j},-1}} \stackrel{k \rightarrow \infty}{\longrightarrow} 0
$$

because $\operatorname{Vol}\left(D_{\zeta^{j_{k},-1}}\right) \rightarrow 0$ by Lemma 4.3. On the other hand

which is a contradiction.

$$
\left\|F_{k}-f\right\|_{D} \geq \frac{\left|f\left(\zeta^{j_{k}}\right)\right|}{K_{D}^{1 / 2}\left(\zeta^{j_{k}}, \zeta^{j_{k}}\right)} \rightarrow 1
$$

5. A counterexample. Let $\Delta$ be the unit disc in $\mathbb{C}$ and define

$$
D_{N}=\Delta \backslash \overline{\bigcup_{k=1}^{\infty} \Delta\left(2^{-k}, 2^{-k N(k)}\right)}
$$

where $\Delta(x, r)$ is the disc centred at $x$ with radius $r>0$. Assume $N(k)>2$ for all $k$. Then $\Delta\left(2^{-k}, 2^{-k N(k)}\right)$ are disjoint from each other for all $k$. We have the following criterion for the hyperconvexity of $D_{N}$ (cf. [12], p. 50):

Proposition 5.1. $D_{N}$ is hyperconvex if and only if $\sum_{k=1}^{\infty} N(k)^{-1}=\infty$.

We first prove the following:

Lemma 5.2. Let $D$ be a bounded domain in $\mathbb{C}$ satisfying:

(*) For each $z_{0} \in \partial D$, there exists a sequence $\left\{z_{k}\right\}_{k=1}^{\infty} \subset \mathbb{C} \backslash \bar{D}$ with $z_{k} \rightarrow z_{0}$ as $k \rightarrow \infty$ such that

$$
\left|z_{k}-z_{0}\right|^{2} \log d\left(z_{k}, \partial D\right) \rightarrow 0 \quad \text { as } k \rightarrow \infty .
$$

Then the Bergman metric on $D$ is complete. 
Proof. First, we prove $K_{D}(z, z) \rightarrow \infty$ as $z \rightarrow \partial D$. For each $z_{0} \in \partial D$, put $f_{k}(z)=\left(z-z_{k}\right)^{-1}$. Then $f_{k}$ is holomorphic on $D$ and satisfies

$$
\left\|f_{k}\right\|_{D}^{2} \leq \int_{D \cap\left\{z|| z-z_{k} \mid>d\left(z_{k}, \partial D\right)\right\}}\left|z-z_{k}\right|^{-2} d V_{1}=2 \pi\left(C_{1}-\log d\left(z_{k}, \partial D\right)\right),
$$

where $C_{1}$ is a constant depending only on the diameter of $D$.

For each $z \in D \cap \Delta\left(z_{0},\left|z_{k}-z_{0}\right|\right)$, one has

$$
K_{D}(z, z) \geq \frac{\left|f_{k}(z)\right|^{2}}{\left\|f_{k}\right\|_{D}^{2}} \geq \frac{1}{8 \pi\left|z_{k}-z_{0}\right|^{2}\left(C_{1}-\log d\left(z_{k}, \partial D\right)\right)} .
$$

Thus we obtain $K_{D}(z, z) \rightarrow \infty$ as $z \rightarrow z_{0}$.

Next we want to show that every $f \in H^{2}(D)$ with $\|f\|_{D} \leq 1$ can be approximated in $L^{2}$-norm by a family $\left\{f_{\varepsilon}\right\}_{\varepsilon>0}$ of holomorphic functions on $D$ which are bounded in some open neighbourhood of $z_{0}$.

For simplicity we can assume $z_{0}=0$ and $D \subset \subset \Delta$. Put

$$
\psi(z)=-\log (-\log |z|) .
$$

Then $\psi$ is a $C^{\infty}$ strictly psh function on $\Delta \backslash\{0\}$ satisfying $\partial \bar{\partial} \psi \geq \partial \psi \bar{\partial} \psi$. For each $0<\varepsilon<1$ we set

$$
\eta_{\varepsilon}=\kappa(-\psi-\log \log (1 / \varepsilon)+1),
$$

where $\kappa$ is the function in the proof of Lemma 4.2. Put

$$
D_{\varepsilon}=\{z \in \mathbb{C}|0<| z \mid<\varepsilon / 2\} \cup D .
$$

Then $\eta_{\varepsilon} f \in C^{\infty}\left(D_{\varepsilon}\right)$. Since every domain in $\mathbb{C}$ is pseudoconvex, we can find a function $y_{\varepsilon}$ on $D_{\varepsilon}$ such that $f_{\varepsilon}=\eta_{\varepsilon} f-y_{\varepsilon}$ is a holomorphic function on $D_{\varepsilon}$ satisfying $\left\|y_{\varepsilon}\right\|_{D_{\varepsilon}} \leq C_{2}\|f\|_{D \cap \Delta\left(0, \varepsilon^{1 / 2}\right)}$, where $C_{2}$ is a constant (independent of $f, \varepsilon)$. This gives

$$
\left\|f_{\varepsilon}-f\right\|_{D} \leq\left(1+C_{2}\right)\|f\|_{D \cap \Delta\left(0, \varepsilon^{1 / 2}\right)}
$$

and $\|f\|_{D_{\varepsilon}} \leq 1+C_{2}$. Then $f_{\varepsilon}$ can be extended to a holomorphic function on $D \cup \Delta\left(0, \varepsilon^{1 / 2}\right)$ and $\left\|f_{\varepsilon}-f\right\|_{D} \rightarrow 0$ as $\varepsilon \rightarrow 0$. The proof of Lemma 5.2 is complete by the remark after Proposition 3.4.

Lemma 5.2 implies

Lemma 5.3. Let $D_{N}$ be defined as above. Suppose that

$$
k N(k) 2^{-2 k} \rightarrow 0 \quad \text { as } k \rightarrow \infty .
$$

Then the Bergman metric on $D_{N}$ is complete.

Now we take $N(k)=k^{2}+1$. Then $D_{N}$ is not hyperconvex by Proposition 5.1 and the Bergman metric on $D_{N}$ is complete by Lemma 5.3.

Acknowledgements. The author wishes to express his most sincere gratitude and appreciation to Professor Jin-Hao Zhang. 


\section{References}

[1] E. Bedford and J. P. Demailly, Two counterexamples concerning the pluricomplex Green function in $\mathbb{C}^{n}$, Indiana Univ. Math. J. 37 (1988), 865-867.

[2] S. Bergman, The Kernel Function and Conformal Mapping, 2nd ed., Amer. Math. Soc., Providence, R.I., 1970.

[3] Z. Błocki, Smooth exhaustion functions in convex domains, Proc. Amer. Math. Soc. 125 (1997), 477-484.

[4] H. J. Bremermann, Holomorphic continuation of the kernel function and the Bergman metric in several complex variables, in: Lectures on Functions of a Complex Variable, Univ. of Michigan Press, 1955, 349-383.

[5] J. P. Demailly, Mesures de Monge-Ampère et mesures pluriharmoniques, Math. Z. 194 (1987), 519-564.

[6] K. Diederich and T. Ohsawa, General continuity principles for the Bergman kernel, Internat. J. Math. 5 (1994), 189-199.

[7] H. Grauert, Charakterisierung der Holomorphiegebiete durch die vollständige Kählersche Metrik, Math. Ann. 131 (1956), 38-75.

[8] L. Hörmander, An Introduction to Complex Analysis in Several Variables, NorthHolland, 1990.

[9] M. Jarnicki and P. Pflug, Bergman completeness of complete circular domains, Ann. Polon. Math. 50 (1989), 219-222.

[10] N. Kerzman et J.-P. Rosay, Fonctions plurisousharmoniques d'exhaustion bornées et domaines taut, Math. Ann. 257 (1981), 171-184.

[11] S. Kobayashi, Geometry of bounded domains, Trans. Amer. Math. Soc. 92 (1959), $267-290$.

[12] T. Ohsawa, On the Bergman kernel of hyperconvex domains, Nagoya Math. J. 129 (1993), 43-52.

[13] _- On the completeness of the Bergman metric, Proc. Japan Acad. Ser. A Math. Sci. 57 (1981), 238-240.

[14] P. Pflug, Various applications of the existence of well growing holomorphic functions, in: Functional Analysis, Holomorphy and Approximation Theory, J. A. Barroso (ed.), North-Holland Math. Stud. 71, North-Holland, 1982, 391-412.

[15] W. Zwonek, On symmetry of the pluricomplex Green function for ellipsoids, Ann. Polon. Math. 67 (1997), 121-129.

Institute of Mathematics

Fudan University

Shanghai 200433

P.R. China

E-mail: 96-165@fudan.edu.cn 